\title{
Electrical properties of macroporous silicon structures
}

\author{
Karachevtseva L.A., Lytvynenko O.A., Malovichko E.A., \\ Sobolev V.D. and Stronska O.J. \\ Institute of Semiconductor Physics, 45 Nauki Prsp., 03028 Kyiv, Ukraine \\ Tel.: 265 9815, Fax: 265 8243, E-mail:kartel@mail.kar.net
}

\begin{abstract}
The dependencies of electron conductivity, concentration and mobility on pore size and concentration were investigated for macroporous silicon structures. The electron conductivity and concentration in two-layer structures of macroporous silicon, and also in a matrix of macroporous layers have a maximum for a macropore volume of $V=0.3-0.4$. Thus the electron mobility decreases monotonically. The experimental results were explained by a model based on the existence of electron-enriched regions around pores, with thickness $\Delta D \approx 1 \mu \mathrm{m}$ formed after electrochemical and chemical treatment of the macropore walls.
\end{abstract}

Keywords: macroporous silicon strucures, electron conductivity, concentration, mobility.

Paper received 26.12.00; revised manuscript received 26.01.01; accepted for publication 16.02.01.

\section{Introduction}

Electrophysical parameters of porous silicon, a prospective material in the field of electrical engineering, have been investigated since the beginning of the $80 \mathrm{~s}$ [1-6]. It has been found that porous silicon layers are highly resistive, with resistances exceeding $10^{5} \Omega \cdot \mathrm{cm}$ for high levels of porosity. A model has been proposed, based on the formation of regions that are depleted by charge carriers due to several factors: capture of carriers by traps on pore surfaces, leaving of extrinsic atoms in an electrolyte and passivation of the impurity atoms by hydrogen. The electrophysical parameters of macroporous silicon structures of $p$-type conductivity were investigated in [7]. The structures were formed in a concentrated solution of hydrofluoric acid under conditions of hole generation by an electric field. The resistivity of the macroporous silicon exceeded the resistivity of the initial silicon by $1.6-15$ times and was determined by the transport of charge carriers through the low resistance regions of the silicon matrix. Over the last few years, research into $n$-type macroporous silicon structures has undergone significant development [8]. Using $n$-Si electrodes, pores of micrometerscale diameters were obtained with a periodical structure $[8,10]$. Thus, the aim of our invstigation was to study the electron conductivity, concentration and mobility in macroporous silicon structures on $n$-Si substrates, taking into account the effect of macropore size and concentration.

\section{Experimental}

The starting material consisted of $n$-type silicon with [100] orientation and 2-5 $\Omega \cdot \mathrm{cm}$ resistivity. Macropores with diameter $D_{p}=1-15 \mu \mathrm{m}$ and depths $h_{p}=50-250 \mu \mathrm{m}$ were formed due to the generation and transfer of nonequilibrium holes to the $n$-Si electrochemically treated surface as a result of optical band-to-band electron-hole generation. The initial $n$-Si plates were chemically polished in a 1:3 $\mathrm{HF}$ and $\mathrm{HNO}_{3}$ solution and anisotropically etched in $10 \%$ solution of $\mathrm{KOH}$ in water to provide pit formation (the periodic structures were anisotropically etched after the photolithographic procedure). Ohmic contacts were made by rubbing some In-Ga eutectic alloy on the edge of the silicon plate and by a platinum electrode in the electrolyte. The sample was mounted in an electrochemical cell and connected to a potentiostat by the 3-electrode scheme. The applied voltage was measured relative to a platinum wire near the sample surface. The electrolyte was 5 weight percent hydrofluoric acid in water. During the electrochemical etching process the sample was illuminated by radiation from a $100 \mathrm{~W}$ tungsten lamp. Additional anisotropy etching in $10 \% \mathrm{KOH}$ solution was used for removing the products of the electrochemical process from the macropore walls.

The microstructure of macropore surfaces was studied by scanning electron microscopy (SEM) using a JEOL JSM-6100 operating at $15 \mathrm{kV}$. The SEM specimens were coated with Au film of about $10 \mathrm{~nm}$ thickness by ion sput- 


\section{L.A. Karachevtseva et al.: Electrical properties of macroporous silicon structures}

tering. The chemical states of the macropore walls were identified by Transmission Fourier Infrared (FTIR) Spectroscopy using the apparatus JEOL JIR-7000. For the infrared analysis the back surface of the samples was mechanically polished to make specimens of $80 \mu \mathrm{m}$ in thickness. To subtract the background absorption of the Si substrate, the same silicon wafer of $80 \mu \mathrm{m}$ thickness was used as a reference.

The $\mathrm{In} / n$-Si and $\mathrm{In} /$ macroporous $n$-Si contacts were formed by thermal evaporation of indium in an atmosphere of hydrogen. The temperature regimes of ohmic contact formation on monocrystalline and on macroporous silicon were comparable with the annealing temperature $T=400^{\circ} \mathrm{C}$ for thermal diffusion of metal through a $\mathrm{SiO}_{\mathrm{x}}$ layer. The contact transient resistance equaled $R_{0} \approx 4-10 \Omega \cdot \mathrm{cm}^{2}$. The electron conductivity, concentration and mobility were measured by the 4-probe method at $300 \mathrm{~K}$.

\section{Results and discussion}

The results of the measurement of the electron conductivity on the macroporous layers were recalculated, taking into consideration the two-layer structure [11] and including the measured parameter $\sigma_{m}$, and the silicon substrate parameters $\sigma_{s}$. The matrix conductivity $\sigma_{p s}{ }^{M}$ of the macroporous layer was determined according to the theory of effective media [11] for cylindrical inclusions. For a comparative analysis of the electron conductivity in the matrix of the macroporous structures the value $\sigma_{p s}{ }^{M}$ was considered relatively to the appropriate silicon substrate parameter $\sigma_{s}$ :

$$
\frac{\sigma_{p s}^{M}}{\sigma_{s}}=\left(\frac{\sigma_{m}}{\sigma_{s}} \frac{H}{h}-\frac{H}{h}-1\right) \frac{1+V}{1-V}
$$

where $H$ and $h$ are the thicknesses of the two-layer structure and of the macroporous silicon layer, respectively; $V$ is the relative pore volume $\left(V=\pi D_{p}^{2} N_{p} / 4, D_{p}\right.$ is a macropore diameter, $N_{p}$ is a macropore concentration).

Fig. 1a shows the dependencies of the relative electron conductivity of the macroporous silicon structures on the macropore volume $V$. The conductivity of electrons in the two-layer structures of macroporous silicon, and also in a matrix of macroporous layers grow with an increase of the macropore volume. These parameters reach a maximum for $V=0.3-0.4$, and then decrease.

It is known that there is an element of error in the electron mobility and concentration recalculation as a result of conductivity and Hall coefficient measurements in nonhomogeneous structures [11]. So we analysed the measured concentration $n_{m}$, and mobility $\mu_{m}$ of electrons taking into account the correlation between recalculated conductivity $\left(\sigma_{p s}{ }^{M}\right)$ and measured conductivity $\left(\sigma_{m}\right)$, as in Fig. 1a.

The electron concentration in the two-layer structures grows with an increase of the macropore volume and reaches a maximum for $V=0.3-0.4$ (Fig. 1b), as electron

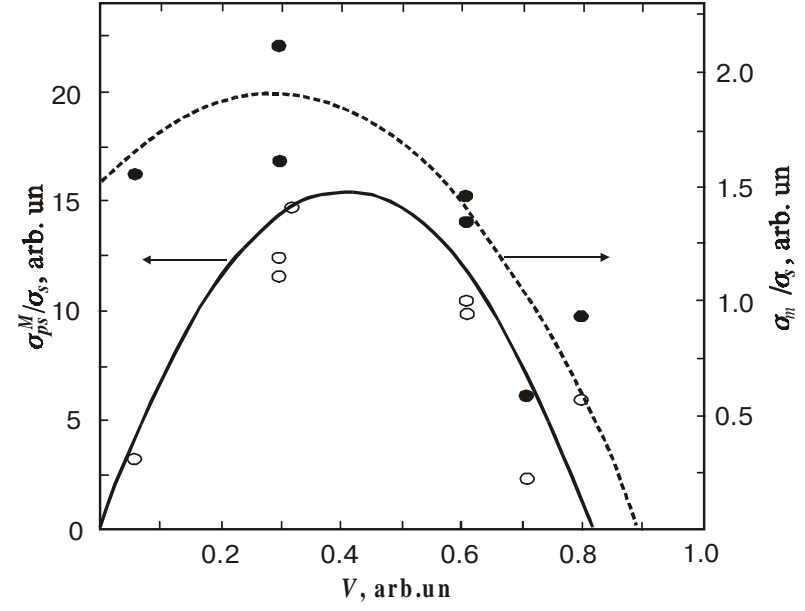

Fig. 1. (a) Relative conductivity of macroporous silicon structures $(\bullet)$ and a matrix of macroporous layers $(O)$ for different values of the macropore volume.

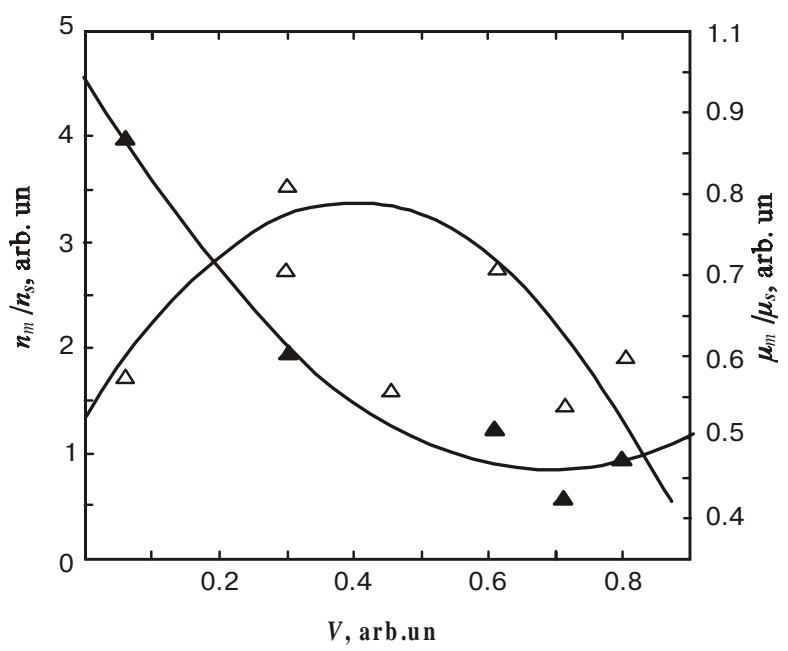

Fig. 1. (b) Relative electron concentration $(\triangle)$ and mobility ( $\Delta$ ) of macroporous silicon structures for different values of the macropore volume.

conductivity. Thus, the electron mobility decreases monotonically.

Figures 2a-c show the dependencies of electron conductivity, concentration and mobility on macropore concentration, diameter and surface. All measured parameters decrease with increase in pore diameter (Fig. 2b), and electron conductivity and concentration increase with increase in pore concentration (Fig. 2a) and surface (Fig. 2c).

An explanation of the experimental results was reached after analysis of the dependencies of electron conductivity, concentration and mobility on the distance between pores $a-D_{p}(a$-average lattice constant of the macroporous structure). Fig. 3a shows such dependencies with a maximum in the electron conductivity and concentration at $a-D_{p} \approx 2 \mu \mathrm{m}$. Therefore, it is possible that regions with thickness $\Delta D \approx 1 \mu \mathrm{m}$, which can be enriched by electrons, can be formed around pores. Fig. $3 \mathrm{~b}$ 
L.A. Karachevtseva et al.: Electrical properties of macroporous silicon structures
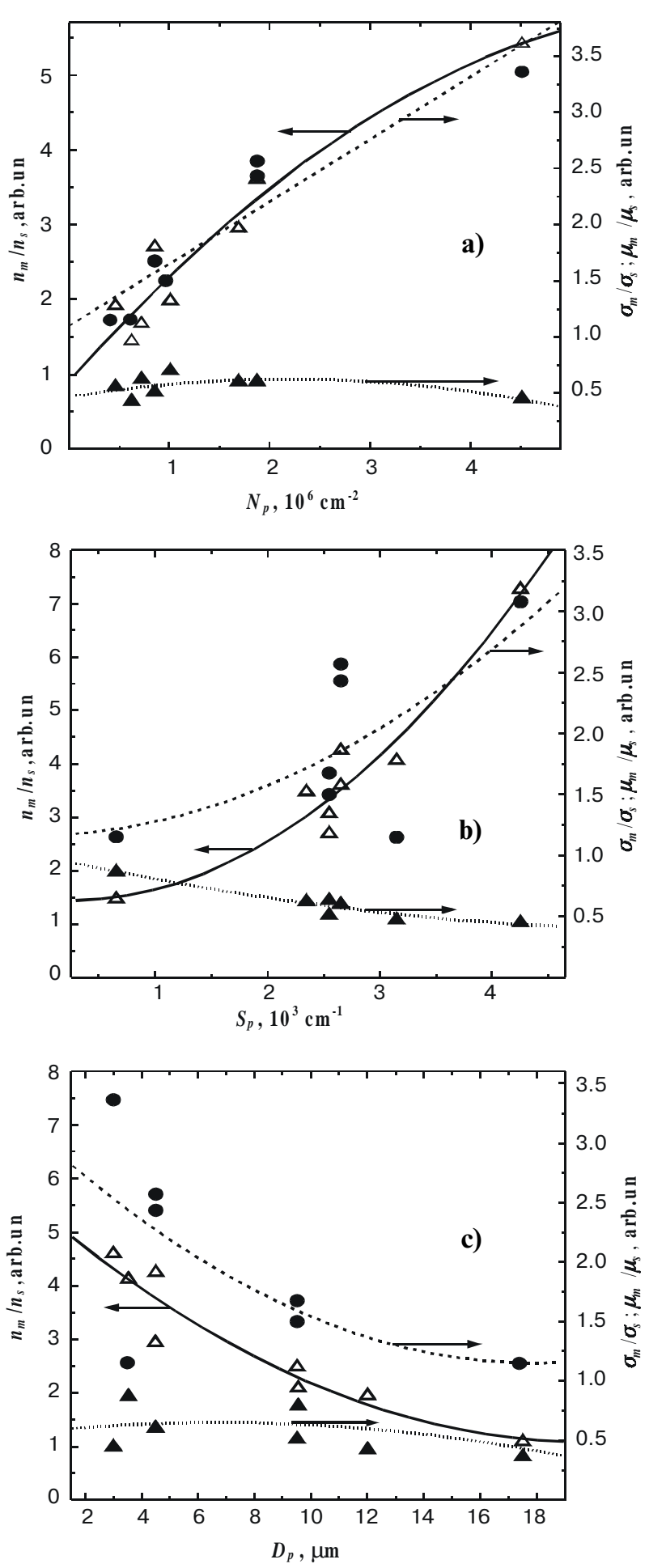

Fig. 2. Dependencies of electron conductivity $(\bullet)$, concentration $(\triangle)$ and mobility $(\Delta)$ in macroporous silicon structures on macropore concentration (a), diameters (b) and surface (c).

shows the dependencies of electron conductivity, concentration and mobility on the volume of the regions $V_{\sigma}$ in macropore structures, reached by electrons:

$V_{\sigma}=\pi \Delta D N_{p}\left(2 D_{p}+\Delta D\right) / 4$.

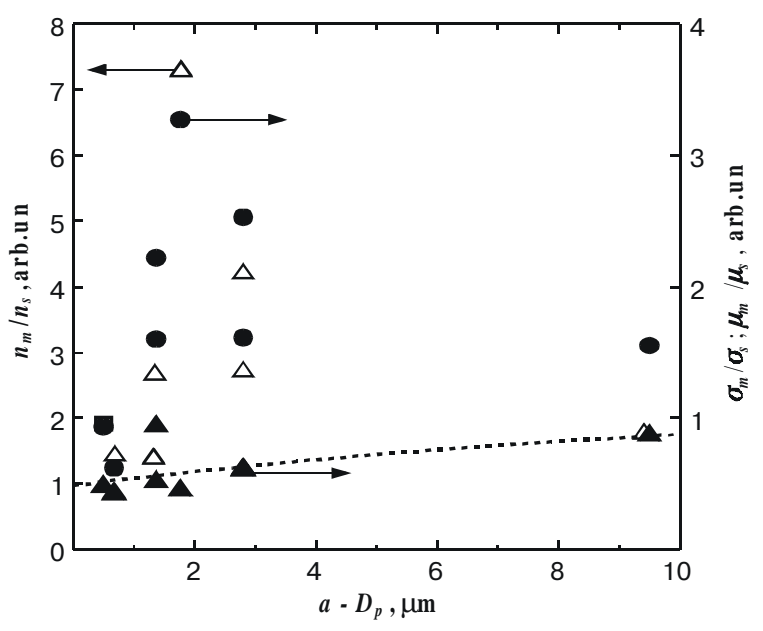

Fig. 3. (a) Dependencies of electron conductivity (•), concentration $(\triangle)$ and mobility $(\mathbf{\Delta})$ in macroporous silicon structures on distance between macropores.

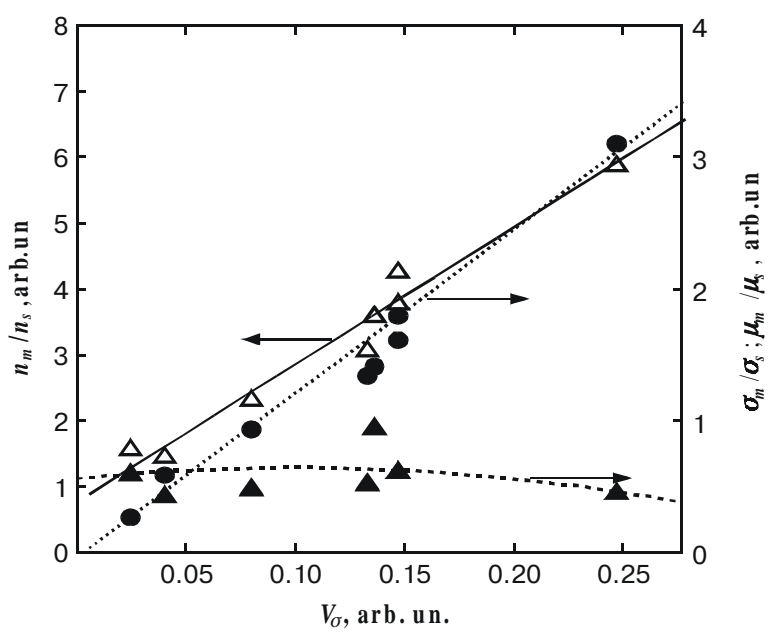

Fig. 3. (b) Dependencies of electron conductivity (•), concentration $(\triangle)$ and mobility $(\mathbf{\Delta})$ in macroporous silicon structures on volume of regions enriched by electrons.

According to Fig. 3a the thickness of regions around pores, enriched by electrons, equals $\Delta D \approx 1 \mu \mathrm{m}$ for $a-D_{p}>2 \mu \mathrm{m}$ and is equal to $\Delta D=\left(a-D_{p}\right) / 2$ for $a-D_{p}<2 \mu \mathrm{m}$. Fig. $3 \mathrm{~b}$ shows the pronounced growth of electron conductivity and concentration with the volume $V_{\sigma}$. This confirms the validity of the model of regions around pores of thickness $\Delta D \approx 1 \mu \mathrm{m}$, enriched by electrons. Really, the measured electron conductivity exceeds the conductivity of the silicon substrate by 2 times, as shown in Fig. 1a; and the electron concentration increases by 4 times in the macroporous structure apparently silicon substrate (Fig. 1b).

The investigated macroporous silicon structures were anisotropically etched after electrochemical treatment, according to the procedures outlined in [8-10] regarding the formation of a macroporous layer. Fig. 4 shows SEM image of macropore wall surfaces before and after $\mathrm{KOH}$ etching. Before $\mathrm{KOH}$ treatment there are pronounced microporous layers around macropores (Fig. 4a, d). Af- 


\section{L.A. Karachevtseva et al.: Electrical properties of macroporous silicon structures}

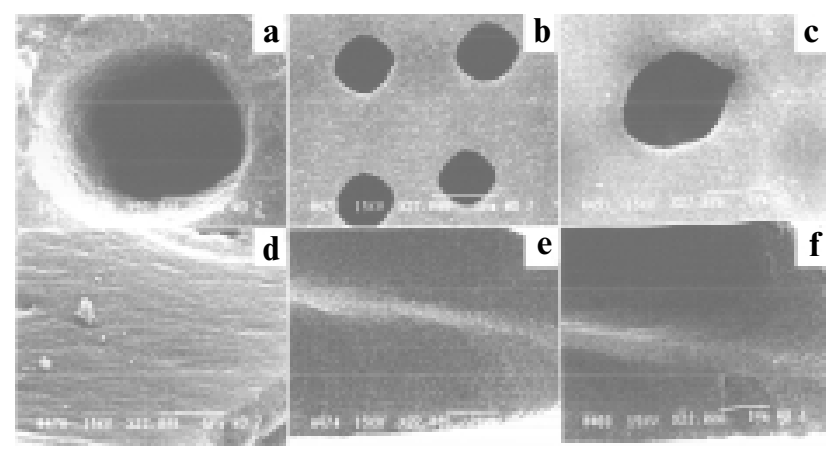

Fig. 4. SEM images of macropore outer surfaces (a)-(c) and macropore walls (d)-(f) of macroporous silicon sample treated with $10 \% \mathrm{KOH}$ for different time; (a) and (d) $0 \mathrm{~s}$, (b) and (e) 10 s, (c) and (f) $20 \mathrm{~s}$.

ter $\mathrm{KOH}$ etching the micropore layer on the macropore surfaces was almost etched off, as in Fig. 4b, c, e, f. Fig. 5 shows typical FTIR spectra of absorption for the macroporous layer before and after etching in $\mathrm{KOH}$. FTIR data indicate that the microporous layer on the macropores contains hydrides, oxides and organic species. After $\mathrm{KOH}$ etching, the FTIR peak intensities decreased. In our case, a high concentration of donor type $\mathrm{OH}^{-}$bonds takes place after removal of the microporous layers from the macropore walls (Fig. 5). So the formation of elec-

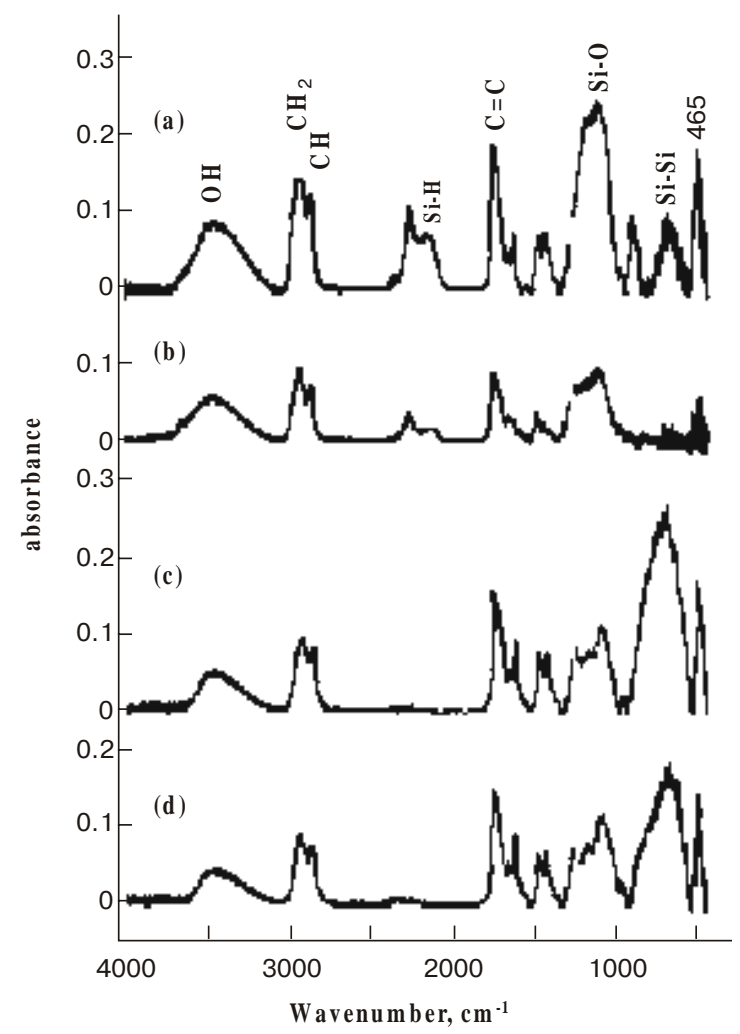

Fig. 5. FTIR spectra of absorption for two macroporous silicon samples, treated with $10 \% \mathrm{KOH}$ for different time; (a) $0 \mathrm{~s}$, (b) $10 \mathrm{~s}$, sample 1; (c) $5 \mathrm{~s}$, (d) $20 \mathrm{~s}$, sample 2 . tron-enriched regions with thickness $\Delta D=1 \mu \mathrm{m}$ is a result of the electrochemical and chemical treatments of macroporous silicon structures.

The model based on the concept of an electron-enriched region explains the experimental dependencies of the electron conductivity and concentration on the macropore volume (Fig. 1a, b). The electron conductivity and concentration increase for samples with a distance between pore walls of $a-D_{p}>2 \mu \mathrm{m}$, have a maximum at $a-D_{p}=2 \Delta D=2 \mu \mathrm{m}$ and then decrease for $a-D_{p}<2 \mu \mathrm{m}$. The enriched region volume $V_{\sigma}$ is proportional to the macropore concentration $N_{p}$, as in Fig. 2a, and Eq. (2).

\section{Conclusions}

The dependencies of electron conductivity, concentration and mobility on pore sizes and concentration have been measured for two-layer macroporous silicon structures. It has been shown that the electron conductivity and concentration in two-layer structures, and also in a matrix of macroporous layers reach a maximum for a macropore volume $V=0.3-0.4$. Thus, the mobility of electrons decreases monotonically. Experimental results were explained by the model of the electron-enriched region around macropore with thickness $\Delta D \approx 1 \mu \mathrm{m}$ formed after electrochemical and chemical treatment of the macropore walls.

\section{References}

1. T. Unagami, Formation mechanism of porous silicon layer by anodisation in HF solution // J. Electrochem. Soc. 127(2), pp. 476-483 (1980).

2. R.C. Anderson, R.S. Muller, C.W. Tobias, Investigation of the Electrical Properties of Porous Silicon // J. Electrochem. Soc. 138(11), pp. 3406-3411 (1991).

3. M.Ben-Chorin, E.Moller and F.Koch, Nonlinear electrical transport in porous silicon // Phys. Rev. B, 49(4), pp. 29812984 (1994).

4. A.J. Simons, T.J. Cox, M.J. Uren, P.D.J. Calcott, The electrical properties of porous silicon producted from $n^{+}$-silicon substrates // Thin solid films 255, pp.12-15 (1995).

5. V.Lehmann, F.Hofmann, F.Möller, U.Grüning, Resistivity of porous silicon: a surface effect // Thin Solid Films 255, pp.2022 (1995).

6. S.P. Zimin, Classification of porous silicon electrical properties // Phizika i Tekhnika Poluprov. 34(3), pp.359-363 (2000) (in Russian).

7. S.P. Zimin. Charge carrier concentration in monocrystal matrix of porous silicon // Letters J. of Tech. Physics 21(24), pp.46-50 (1995) (in Russian).

8. V. Lehmann, H. Foll, Formation Mechanism and Properties of Electrochemically Etched Trenches in $n$-Type Silicon // $J$. Electrochem. Soc. 137(2), pp. 653-659 (1990).

9. L.A. Karachevtseva, O.A. Lytvynenko, O.J. Stronska, Development and optical characteristics of the macroporous silicon structures // Semiconductor Physics, Quantum Electronics and Optoelectronics 3(1), pp.22-25 (2000)

10. L.A. Karachevtseva, O.A. Lytvynenko, E.A. Malovichko, O.J. Stronska, Investigation of Electrochemical Formation of Macropores in Silicon // Theor. and Experim. Chem. 36(3), pp.193-197 (2000).

11. E.V. Kuchis, Methods of Hall Effect Investigation, Sov. Radio, Moscow (1974), 328 p. (in Russian). 